\title{
Cambios en rasgos funcionales de las hojas de Piper reticulatum (Piperaceae) en luz y sombra en La Selva, Costa Rica
}

\author{
Daniel Rodríguez García1 (iD), Camila Delgado Montes² (D), Yitzendi López Serrano³ (iD), \& Rosa Brooks \\ Laverdeza 4 iD \\ 1. Universidad Nacional de Costa Rica, Avenida 1, Calle 9, Heredia, Costa Rica; drodrig44p@gmail.com \\ 2. Vassar College, 124 Raymond Ave, Poughkeepsie, NY 12604, Estados Unidos; cadelgadomontes@gmail.com \\ 3. Instituto de Ecología, A. C, Carretera antigua a Coatepec 351, El Haya, Xalapa 91070, Veracruz, México; \\ yitzendi.lopez@posgrado.ecologia.edu.mx \\ 4. Centro Oriental de Ecosistemas y Biodiversidad, Santiago de Cuba, Cuba; rosambrookslaverdeza@gmail.com
}

\author{
Recibido 27-IX-2019 • Corregido 20-I-2020 • Aceptado 28-II-2020
}

DOI: https://doi.org/10.22458/urj.v12i1.2799

\begin{abstract}
Changes in functional traits of Piper reticulatum (Piperaceae) leaves under light and shade in La Selva, Costa Rica". Introduction: Plants are exposed to environments with different light availability, and light is the major limiting factor in tropical rainforests. In response, leaves modify their functional traits to exploit available light and avoid hydric stress. However, how plants change their functional traits in different light conditions remains poorly understood. Objective: This study concentrates on the adaptation of Piper reticulatum (Piperaceae) leaves in different light environments. Additionally, the study investigates whether these changes occur in the leaves' young or mature stages. Methods: Sixty leaves (one young and one mature per individual) were collected from a total of $30 \mathrm{P}$. reticulatum individuals in La Selva Biological Station, Costa Rica, 15 individuals were in the shade and 15 individuals were exposed to direct sunlight. The leaf area and specific leaf mass of each leaf was measured to record differences in biomass allocation. Additionally, the stomata and tertiary veins of each leaf were counted to determine whether there are adaptations against dehydration in environments with more light. Results: Sunlit leaves had a higher number of stomata, fewer tertiary veins, and higher specific leaf mass. Furthermore, younger leaves showed more stomata but lower specific leaf mass. Conclusions: Plants under different light conditions differed in stomata, veins and mass, and leaf age was a factor.
\end{abstract}

Keywords: Leaf area, ontogeny, stomas, specific leaf mass, plasticity.
RESUMEN. Introducción: Las plantas están expuestas a ambientes con diferente disponibilidad de luz, la cuál es el factor más limitante en los bosques tropicales húmedos. En respuesta, las hojas modifican sus rasgos funcionales para aprovecharla y evitar el estrés hídrico. Sin embargo, aún falta información para comprender cómo la planta cambia los rasgos funcionales dependiendo de las condiciones lumínicas. Objetivos: Por ello, este estudio se concentró en la adaptación de las hojas de Piper reticulatum (Piperaceae) ante diferentes ambientes de luz. Además, se quiso conocer si estos cambios ocurren en etapas jóvenes o maduras de la hoja. Métodos: Se recolectaron sesenta hojas (una joven y una vieja) de $P$. reticulatum en 15 individuos que se encontraban en la sombra y 15 individuos expuestos al sol en la Estación Biológica La Selva, Costa Rica. Se midió el área foliar y la masa foliar específica de cada hoja para determinar diferencias en la asignación de biomasa. También, se contabilizaron los estomas y las venas terciarias para determinar si hay adaptaciones contra la desecación en ambientes de mayor cantidad de luz. Resultados: Las hojas de sol tuvieron mayor cantidad de estomas, menos venas terciarias y mayor masa foliar específica. También, las hojas jóvenes presentaron más estomas, pero menos masa foliar específica. Conclusión: Plantas bajo diferentes condiciones de luz, difieren en cantidad de estomas, venas y masa, siendo la edad de la hoja un factor de variación.

Palabras clave: Área foliar, ontogenia, estomas, masa foliar específica, plasticidad. 
Los bosques son ambientes heterogéneos de luz, temperatura y humedad debido a los cambios en la cobertura vegetal del dosel. Para adaptarse a estas condiciones, las plantas modifican características morfológicas, bioquímicas y en la regeneración de tejidos. Así aprovechan recursos limitantes como la luz, que utilizan para su crecimiento y supervivencia (Markesteijn, Poorter, \& Bongers, 2007). Estas modificaciones se denominan rasgos funcionales y dependen del entorno físico y biótico (de Bello et al., 2010).

Generalmente, las hojas bajo alta incidencia lumínica tienen una menor área foliar y un mesófilo más desarrollado. Esto aumenta su capacidad fotosintética y ayuda a proteger a la hoja contra la saturación lumínica (Terashima, Hanba, Tazoe, Vyas, \& Yano, 2005). Igualmente, en ambientes de alta radiación solar, las plantas suelen aumentar la cantidad de estomas, los cuales tienen la capacidad de modificar su apertura con altas concentraciones de $\mathrm{CO}_{2}$ (Hetherington \& Woodward, 2003). Además, una mayor cantidad de venas en la hoja contribuye a minimizar la deshidratación de la planta en ambientes con luz porque transportan agua y derivados de la fotosíntesis (Sack \& Scoffoni, 2013). Estas adaptaciones estructurales permiten un mejor desempeño de la planta en condiciones cambiantes, lo que aumenta su probabilidad de supervivencia y mejora su competencia inter e intraespecífica.

Aunque los rasgos funcionales varían según las condiciones ambientales presentes, también podrían hacerlo por la ontogenia de la hoja (Pérez-Harguindeguy et al., 2013). Se ha encontrado que las hojas maduras y jóvenes responden de manera diferente a la luz (Tafolla-Arellano et al., 2013). Algunos estudios demuestran que las hojas jóvenes son menos efectivas en el proceso de fotosíntesis que las hojas maduras, pero que están mejor adaptadas a condiciones extremas (Shirke, 2001; Pérez-Harguindeguy et al., 2013). Sin embargo, se desconoce en cuál etapa de la hoja ocurren estos cambios en respuesta a la disponibilidad de luz.

En este trabajo se quiso probar la variación de los rasgos funcionales de la hoja de la planta Piper reticulatum (Piperaceae) en respuesta a cambios en las condiciones de luz, y según la edad de la hoja. Se espera que las hojas expuestas a la luz tengan más estomas, venas terciarias y masa foliar específica pero menor área foliar porque no necesitan maximizar la captura de energía lumínica. Además, se quiere determinar si estos rasgos son diferentes en hojas jóvenes y hojas viejas, lo cual puede ser un indicador de que los cambios en los rasgos funciones se muestran en etapas tempranas del desarrollo de la hoja.

\section{MATERIALES Y MÉTODOS}

La recolección de datos se desarrolló en la Estación Biológica La Selva, Sarapiquí, Costa Rica, la cual recibe una precipitación anual promedio de $4000 \mathrm{~mm}$ y su vegetación corresponde a Bosque Muy Húmedo Tropical según la clasificación de Zonas de Vida de Holdridge (Holdridge, 1967). Se utilizó la planta Piper reticulatum (Piperaceae) por su abundancia y porque tolera ambientes tanto de sol como de sombra (Villegas \& Chavarría, 2016). Con un densiómetro, se estimó la cobertura vegetal circundante y se seleccionaron 30 individuos, 15 en condiciones de luz (entre $35 \%$ y $75 \%$ de cobertura vegetal) y 15 en sombra (más del $80 \%$ de cobertura vegetal). Por cada individuo se recolectaron dos hojas: una cercana al meristemo apical, que se denominó hoja joven, y otra a tres hojas debajo de esta, la cual correspondió a la hoja vieja.

Se estimó el área foliar, la cantidad de estomas, la cantidad de venas terciarias y la masa foliar específica de cada hoja. El área foliar se calculó utilizando un medidor de área foliar (LI3100C, LI-COR, Lincoln Nebraska, USA). La cantidad de estomas se determinó contando las estructuras visibles en el área de visión de un lente de microscopio 40X. La cantidad de venación terciaria se calculó promediando el número de venas terciarias que interceptaban tres líneas de $1 \mathrm{~cm}$ de largo hechas en la base, centro y ápice de la hoja. Por último, se determinó la masa foliar específica 
dividiendo el peso seco de la hoja en un centímetro cuadrado, según la metodología propuesta por Pérez-Harguindeguy et al. (2013).

Para el análisis estadístico, se realizaron modelos lineales para caracterizar el área foliar de la hoja, la cantidad de estomas y la cantidad de venas terciarias de las hojas, en función de la edad de la hoja, la disponibilidad de luz y la interacción entre estos dos factores. Todos los análisis fueron realizados con el programa $R$, versión 3.5.1 ( $R$ Core Team, 2018).

Ética, conflicto de intereses y declaración de financiamiento: Los autores declaran haber cumplido con todos los requisitos éticos y legales pertinentes, tanto durante el estudio como en el manuscrito; que no hay conflictos de interés de ningún tipo, y que todas las fuentes financieras se detallan plena y claramente en la sección de agradecimientos. Asimismo, están de acuerdo con la versión editada final del documento. El respectivo documento legal firmado se encuentra en los archivos de la revista.

\section{RESULTADOS}

El área foliar de las hojas no presentó diferencias según la cobertura $(F=0,18, g l=58, p=$ $0,67)$ y tampoco con la edad de la hoja $(F=0,33, g l=58, p=0,56)$. La cantidad de estomas fue mayor en hojas jóvenes $(F=-2,22, g l=60, p=0,030)$, y en ambientes de sol $(F=-4,25, g l=60, p<0,001)$. La venación de las hojas fue mayor en ambientes con más cobertura ( $F=3,49, g l=1, p<0,001)$, pero no presentó diferencias según la edad $(F=-0,199, g l=1, p=0,84)$. Por último, la masa foliar específica de las hojas fue significativamente mayor en hojas viejas que en hojas jóvenes $(F=11,13, g l=58, p<$ $0,001)$. También, la masa foliar específica de las hojas fue mayor en condiciones de sol que de sombra ( $F=13,33, g l=58, p<0,001)$ (Fig. 1.).
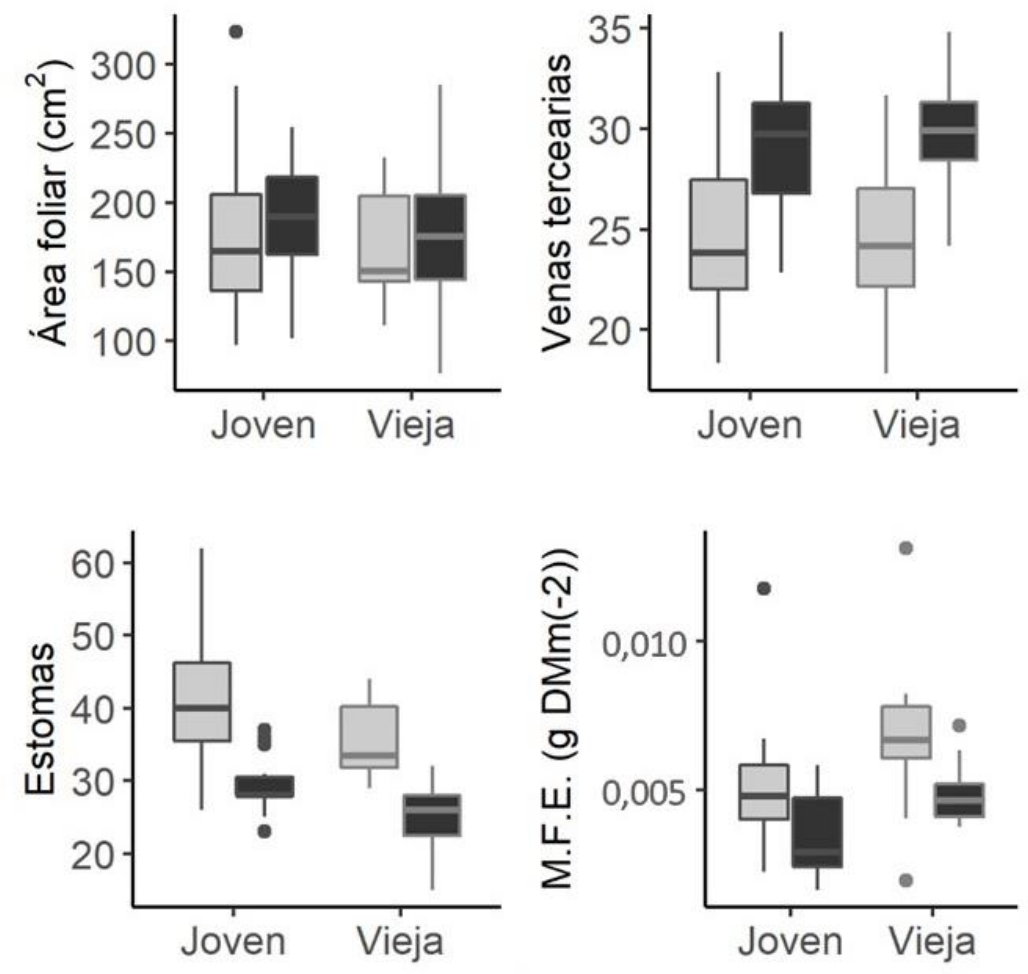

Edad de la hoja

Fig. 1. Diferencias en los rangos funcionales según la edad de la hoja (joven y vieja) y la disponibilidad de luz en dos categorías: sol (en gris) y sombra (en negro). 


\section{DISCUSIÓN}

En este estudio se quiso determinar si el área foliar, la cantidad de estomas, la cantidad de venas terciarias y la masa foliar específica son afectados por la incidencia de luz, la ontogenia de la hoja o ambas. Los resultados mostraron que la disponibilidad de luz no genera un efecto significativo en el área foliar de las hojas de $P$. reticulatum. Esto difiere con los planteamientos de Lusk (2002) y Villegas-Retana y Chavarría-Soto (2016) al encontrar mayor área foliar en hojas $P$. reticulatum expuestas al sol. Sin embargo, puede deberse a diferencias en las metodologías empleadas, porque el presente estudio utilizó una distinción clara entre ambientes de sol y sombra, lo cual no se aplicó en las investigaciones antes mencionadas.

Contrario a nuestra predicción, los estomas fueron más abundantes en ambientes de sol que de sombra. Esto posiblemente se explique debido a que la tasa fotosintética es mayor en las hojas expuestas al sol; por ello, se necesita mayor cantidad de estructuras (como estomas) que ayuden al intercambio gaseoso (Hetherington \& Woodward, 2003). Al igual que en las hojas de este estudio, se ha encontrado menor cantidad de estomas en hojas de helechos que están en vegetación espesa, es decir en condiciones de sombra, donde no están expuestas a la radiación directa (Allard, Nelson, \& Pallardy, 1991). Es posible que la reducción del número de estomas en estos ambientes sea una adaptación de diferentes taxones a la sombra, y no solamente de las plantas vasculares.

Aunque se esperaba que hubiera más abundancia de venas terciarias en las hojas expuestas al sol debido a la necesidad de un suministro constante de agua, los resultados demuestran que hay más venas terciarias en hojas que experimentan poca radiación solar. Esto puede explicarse desde el punto de vista estructural, ya que hojas más grandes, que generalmente son las de sombra, necesitan un peciolo más largo y mayor cantidad de venas para sostenerse. Además, el transporte eficiente de agua y azúcares necesita más vascularización debido a su baja tasa fotosintética (Sack \& Scoffoni, 2013).

En cuanto al efecto de la ontogenia de la hoja, se detectó que las hojas viejas y expuestas al sol son más pesadas que las hojas nuevas y en sombra. Esto confirma la predicción realizada y se explica porque las hojas cercanas al meristemo apical de la rama, no han desarrollado tanto material vegetal. Esto provoca que las hojas sean generalmente más blandas y poco pesadas (PérezHarguindeguy et al., 2013; Tafolla, González, García \& Báez, 2013). También Avalos y Mulkey (2004) y Castrillo, Vizcaíno, Moreno y Latocarra (2005) observaron esta característica en especies de la familia Lamiaceae relacionándolo con la protección contra la herbívora y disminución de la fotoinhibición de la clorofila.

Este trabajo profundizó en los rasgos funcionales de la planta Piper reticulatum que varían en ambientes de sol y sombra. Los resultados demuestran que hay aclimataciones (mayor número de estomas, menos venas terciarias y mayor masa foliar específica) de las plantas a las condiciones ambientales a nivel de hoja. La edad es un factor que afecta a la mayoría de los rasgos funcionales que están asociados al desarrollo de la hoja, excepto al área foliar, la cual no varía entre hojas viejas y jóvenes.

\section{AGRADECIMIENTOS}

Agradecemos a la Organización para Estudios Tropicales (OET). A Sofía Rodríguez Brenes, Fernando Soley y Eduardo Chacón por su asesoría y apoyo durante la elaboración de este artículo. 


\section{REFERENCIAS}

Allard, G., Nelson, C. J., \& Pallardy, S. G. (1991). Shade Effects on Growth of Tall Fescue: I. Leaf Anatomy and Dry Matter Partitioning. Crop Science, 31, 163-167. DOI: 10.071/PP00157

Avalos, G., \& Mulkey, S. (2004). Photosynthetic acclimation of the liana Stigmaphyllon lindenianum to light changes in a tropical dry forest canopy. Oecologia, 120, 475-484. DOI: 132.203.227.63

Castrillo, M., Vizcaíno, D., Moreno, E., \& Latorraca, Z. (2005). Specific leaf mass, fresh: dry weight ratio, sugar and protein contents in species of Lamiaceae from different light environments. Revista de Biología Tropical, 53, 23-28.

de Bello, F., Lavorel, S., Díaz, S., Harrington, R., Cornelissen, J. H., Bardgett, R. D., \& da Silva, P. M. (2010). Towards an assessment of multiple ecosystem processes and services via functional traits. Biodiversity and Conservation, 19 (10), 2873-2893. DOI: 10.1007/s10531-010-9850-9

Hetherington, A. M., \& Woodward, F. I. (2003). The role of stomata in sensing and driving environmental change. Nature, 424 (6951), 901. DOI: 10.1038/nature01843

Holdridge, L. R. (1967). Life Zone Ecology. San Jose, Costa Rica: Tropical Science Center.

Lusk, C. H. (2002). Leaf area accumulation helps juvenile evergreen trees tolerate shade in a temperate rainforest. Oecologia, 132, 188-196. DOI: 10.1007/s00442-002-0974-9

Markesteijn, L. Poorter, L., \& Bongers, F. (2007). Light-Dependent leaf trait variation in 43 tropical dry forest tree species. Americal Journal of Botany, 94(4), 515-525. DOI: 10.3732/ajb.94.4.515

Pérez-Harguindeguy, N., Díaz, S., Garnier, E., Lavorel, S., Poorter, H., \& Jaureguiberry, P. (2013). New handbook for standardised measurement of plant functional traits worldwide. Australian Journal of Botany, 61, 167-234. DOI: 10.1071/BT12225

R Core Team. (2018). R: A language and environment for statistical computing. R Foundation for Statistical Computing, Vienna, Austria. Recuperado de https://www.R-project.org/.

Sack, L., \& Scoffoni, C. (2013). Leaf venation: structure, function, development, evolution, ecology and applications in the past, present and future. New Phytologist, 198(4), 983-1000. DOI: 10.1111/nph.12253

Shirke, P. A. (2001). Leaf photosynthesis, dark respiration and fluorescence as influenced by leaf age in an evergreen tree, Prosopis juliflora. Photosynthetica, 39(2), 305-311. DOI: 10.1023/A:1013761410734

Tafolla-Arellano, J. C., González-León, A., Tiznado-Hernández, M. E., Zacarías García, L., \& Báez-Sañudo, R. (2013). Composición, fisiología y biosíntesis de la cutícula en plantas. Revista Fitotecnia Mexicana, 36(1), 3-12. Recuperado de http://www.scielo.org.mx/scielo.php?script=sci_arttext\&pid=S0187-73802013000100001\&lng=es\&tlng=en.

Terashima, I., Hanba, Y. T., Tazoe, Y., Vyas, P., \& Yano, S. (2005). Irradiance and phenotype: comparative eco-development of sun and shade leaves in relation to photosynthetic $\mathrm{CO}_{2}$ diffusion. Journal of Experimental Botany, 57(2), 343-354. DOI: 10.1093/jxb/erj014

Villegas, S. A., \& Chavarría, M. (2016). Leaf morphology and chlorophyll in Piper reticulatum (Piperaceae) under conditions of light and shadow at La Selva Biological Station, Costa Rica. UNED Research Journal, 8(2), 255-258. DOI: 10.22458/urj.v8i2.1569 